\title{
Le nouveau programme de résidence de médecine familiale de l'Université de Montréal : des intentions aux premiers pas de l'évaluation
}

\section{The new family medicine residency program of the University of Montreal: from intentions to the onset of evaluation}

\author{
Gilbert SANCHE, Louise AUTHIER, Caroline BË̈QUE et Réjean DUPLAIN \\ Programme de résidence de médecine familiale, Faculté de médecine, Université de Montréal, Québec, Canada
}

Manuscrit reçu le 4 mai 2009 ; commentaires éditoriaux formulés aux auteurs le 22 juin 2009 et le 6 février 2010 ; accepté pour publication le 10 février 2010

\begin{abstract}
Mots clés :
Enseignement ; pédagogie médicale ; résidence ; médecine familiale ; médecine générale ; formation médicale ; évaluation

Keywords:

Teaching;

medical teaching; residency program; curriculum revision; family medicine; primary care medicine; program evaluation
\end{abstract}

\begin{abstract}
Résumé - Contexte : En 1998, le directeur du département de médecine familiale confiait à un Comité de révision du curriculum le mandat de revoir le programme de résidence en médecine familiale de l'Université de Montréal. Le rapport de ce comité a servi de base à une ambitieuse réforme du programme, entreprise en 2006, visant à améliorer la formation des futurs médecins de famille pour qu'ils puissent répondre encore plus adéquatement aux besoins de santé de la population en œuvrant dans un réseau de santé en transformation. Buts et méthodes : En expliquant les raisons et les modalités de révision d'un programme de résidence, les auteurs proposent une réflexion et une démarche généralisables pour tous les départements de médecine générale qui souhaitent repenser leur curriculum. Messages clés : L'article expose successivement le contexte académique et professionnel de la démarche, la problématique et les objectifs poursuivis, les orientations retenues pour le nouveau programme, le processus de révision curriculaire mis en œuvre, les caractéristiques du nouveau programme et les résultats d'une évaluation préliminaire.
\end{abstract}

Abstract - Context: In 1998, the department of Family Medicine of the University of Montreal proceeded to the revision of it's residency curriculum. The report of the Revision Committee was the starting point of an ambitious reform of the residence program meant to improve the education and training of the future family physicians. Goals and methods: By explaining the raisons for, and the means by which the curriculum was modified, the authors are proposing a reflection and a method that could provide inspiration to family medicine programs that would like to get involved in such a process. Key messages: The article explains how the new family medicine residency program of the University of Montreal was conceived from the identification of the health needs of the population to the first step of it's evaluation, emphasizing the guiding educational principles and the tool specifically designed to facilitate the implementation. The authors will conclude by addressing the challenges that still confront both the faculty and residents. 


\section{Introduction}

La contribution qui suit rapporte une ambitieuse réforme du programme de résidence en médecine familiale de l'Université de Montréal, entreprise en 2006 sur la base d'un rapport établi par un comité de révision du curriculum, mis en place en 1998. Le but est d'améliorer la formation des futurs médecins de famille pour qu'ils puissent répondre encore plus adéquatement aux besoins de santé de la population en œuvrant dans un réseau de santé en transformation. En rendant explicites les raisons et les modalités de révision de ce programme, notre intention est de proposer une réflexion et une démarche généralisables pour tous les départements de médecine générale qui souhaitent repenser le curriculum de leur programme post-gradué en médecine générale ou médecine de famille. Nous exposerons successivement le contexte académique et professionnel de la démarche, la problématique et les objectifs poursuivis, les orientations retenues pour le nouveau programme, le processus de révision curriculaire mis en œuvre, les caractéristiques du nouveau programme et les résultats d'une évaluation préliminaire.

\section{Contexte académique et professionnel}

En 1998, le directeur du département de médecine familiale de l'Université de Montréal formait un groupe de travail, nommé Comité de révision du curriculum (CRC), en lui donnant le mandat de réviser son programme de résidence. Cette décision n'était pas motivée par la «performance » du programme qui, cette année-là, venait d'obtenir un agrément complet du Collège des médecins de famille du Canada (CMFC) et du Collège des médecins du Québec (CMQ) et dont les étudiants finissants, année après année, obtenaient à l'examen terminal de ces deux mêmes collèges des résultats comparables à ceux des résidents des autres programmes du Québec et du Canada. L'exercice de révision était plutôt entrepris en lien avec le questionnement que suscitait alors la mise sur pied, partout au Canada, de programmes de formations complémentaires aux deux années de la résidence de médecine familiale. Ces programmes, d'une durée de quelques mois à une année, très populaires auprès des résidents, proposaient des formations dans plusieurs domaines : soins d'urgence, santé mentale, soins palliatifs, médecine rurale, périnatalité, médecine du sport, santé des populations, soins aux personnes âgées, etc. En 1998, 18 \% des finissants de notre programme s'inscrivaient dans l'une des trois formations que nous offrions alors à Montréal : soins d'urgence, soins aux personnes âgées et soins palliatifs. À l'échelle du Canada, la section des résidents du CMFC et l'ensemble des directeurs de programme de médecine familiale canadiens estimaient que $40 \%$ de la cohorte des étudiants finissants souhaitaient accéder à de tels programmes complémentaires. Le projet de révision de notre curriculum a donc été suscité, d'une part par la prise en compte d'un besoin de formation ressenti par un groupe significatif d'étudiants finissants et de leur désir de prolonger leur résidence et, d'autre part, par le besoin de vérifier si un programme de résidence datant de plus de vingt ans et n'ayant jamais été révisé de façon globale était encore en mesure de former adéquatement les médecins de famille pour faire face aux nouveaux défis auxquels le $\mathrm{XXI}^{\mathrm{e}}$ siècle allait les confronter, dans une société qui réorganisait ses modes de prestation de soins.

Au terme de ses travaux, en 2001, le CRC a abouti à la conclusion que le programme ne répondait plus de façon optimale aux besoins de formation d'un médecin de famille que l'on voulait compétent, professionnel, polyvalent, capable d'œuvrer partout au Québec, principalement en première ligne. S'appuyant sur plusieurs constats (tableau I), son rapport $^{[1]}$ se concluait par une série de recommandations. La première d'entre elles prônait de passer d'un cursus de deux ans à un cursus de trois ans. Conscient du temps que demanderait l'application de cette recommandation, le CRC préconisait, entre temps, de modifier le programme existant pour que la nouvelle formation : permette aux futurs médecins de famille de mieux répondre aux besoins de santé de la population du Québec ; privilégie comme 
Tableau I. Constats du rapport du Comité de révision du programme de résidence en médecine familiale.

La popularité des formations complémentaires témoigne de la difficulté qu'a notre programme, dans son cadre et sa durée actuels, à former des médecins réellement aptes à exercer la pratique polyvalente de la médecine familiale.

L'exposition à la médecine générale, limitée à 224 jours, est insuffisante. Comparativement, les programmes de médecine familiale américains offrent à leurs résidents une exposition qui varie de 272 à 365 jours.

La succession de stages concentrés, réalisés majoritairement auprès de spécialistes en milieu hospitalier, propose un modèle de pratique qui diffère de celui d'un médecin de famille en exercice, limite l'exposition à la première ligne et complique la continuité des soins que les résidents doivent offrir à leur clientèle de patients.

Avec moins de $10 \%$ de temps de formation laissé au choix des résidents, le programme manque de souplesse en laissant trop peu de possibilités aux résidents d'apporter une coloration personnelle à leur formation afin de répondre aux besoins qu'ils ressentent et identifient.

Plusieurs stages ont un faible rendement pédagogique et bénéficieraient d'une restructuration, parfois majeure. L'intégration de ces stages dans une structure longitudinale permettrait de mieux atteindre certains objectifs d'apprentissage.

La multiplication d'activités d'enseignements théoriques qui ne sont pas regroupées en un programme formel, commun à toutes les unités de médecine de famille, s'est faite au détriment de l'apprentissage expérientiel clinique.

Des carences spécifiques sont identifiables dans l'enseignement de la santé mentale, du locomoteur et de la pharmacothérapie.

moyen d'apprentissage une exposition clinique pertinente pour un médecin de famille; se déroule principalement au sein d'unités de médecine familiale (UMF) appelées à se transformer en milieux modèles de pratique clinique, davantage orientés vers la communauté et bien intégrés dans le réseau de soins de santé ; corrige certaines lacunes spécifiquement identifiées et notamment l'enseignement des problèmes de santé concernant l'appareil locomoteur, de la pharmacothérapie et de la santé mentale.

De 2001 à 2004, la direction du département a entrepris les démarches nécessaires pour obtenir l'autorisation de réaliser un programme de trois ans. Bien qu'accepté au niveau universitaire, le projet d'un tel programme s'est cependant heurté à une fin de non-recevoir au niveau politique, en raison de la pénurie de médecins qui sévit au Québec (l'allongement de la résidence aurait, bien sûr, eu pour effet de priver la population d'une cohorte de nouveaux médecins pendant une année, ce qui était inacceptable aux yeux de certains) et d'un doute quant à la nécessité de prolonger la résidence. Dès lors, le défi d'avoir à corriger et à développer le programme à l'intérieur d'un cadre temporel restreint à deux ans a imposé de repenser tant les options pédagogiques que les structures d'un nouveau cursus. La direction du programme de résidence a alors formé un Comité du renouveau de la résidence $(\mathrm{CReR})$ qui s'est employé, de 2004 à 2006, à concevoir et à faire adopter par les instances départementales puis facultaires un nouveau programme de résidence respectant le cadre imposé de deux ans.

C'est en juillet 2006 qu'ont débuté les travaux d'implantation de ce nouveau programme. Les 14 UMF affiliées à l'Université de Montréal, qui constituent le port d'attache clinique et pédagogique des résidents durant les deux années de leur formation, ont alors reçu le mandat d'évoluer en trois ans, à leur rythme et en fonction de leurs ressources, de l'ancien vers le nouveau programme.

\section{Problématique et objectifs}

Dans sa démarche de planification du nouveau programme, le CReR a d'abord considéré les principaux besoins de santé de la population auxquels les futurs médecins de famille allaient devoir répondre. Ces besoins avaient déjà été bien identifiés par les nombreuses consultations menées par le 
Tableau II. Principes, prémisses et applications pédagogiques du nouveau programme.

\begin{tabular}{|c|c|c|}
\hline Les principes & Les prémisses & Les applications \\
\hline La signifiance identitaire & $\begin{array}{l}\text { Une exposition à des tâches authentiques, } \\
\text { complexes et signifiantes, en plus de stimu- } \\
\text { ler la motivation à apprendre, favorise la } \\
\text { construction de l'identité professionnelle et } \\
\text { facilite le développement d'un sentiment de } \\
\text { compétence. } \\
\text { Le médecin de famille enseignant assure la } \\
\text { pertinence de l'exposition clinique et joue le } \\
\text { rôle de modèle inspirant. }\end{array}$ & $\begin{array}{l}\text { Le travail en résidence s'apparente à } \\
\text { celui du médecin de famille en exer- } \\
\text { cice. } \\
\text { La plus grande partie de l'enseigne- } \\
\text { ment est assumée par les médecins de } \\
\text { famille. }\end{array}$ \\
\hline L'itération & $\begin{array}{l}\text { L'itération est un moyen privilégié pour fa- } \\
\text { voriser l'apprentissage en profondeur et l'in- } \\
\text { tégration des composantes des compétences. }\end{array}$ & $\begin{array}{l}\text { L'exposition clinique répétée dans les } \\
\text { principaux domaines d'apprentissages } \\
\text { est répartie sur deux ans plutôt que } \\
\text { concentrée par stages de quatre se- } \\
\text { maines qui ne se répètent pas. }\end{array}$ \\
\hline La responsabilisation & $\begin{array}{l}\text { L'exercice supervisé d'une pratique authen- } \\
\text { tique stimule les résidents à développer da- } \\
\text { vantage leur sens de responsabilité et leur } \\
\text { professionnalisme, qui se manifesteront par } \\
\text { un engagement soutenu à offrir des soins } \\
\text { globaux et continus à une clientèle définie } \\
\text { de patients. }\end{array}$ & $\begin{array}{l}\text { Suivi de sa propre clientèle de patients } \\
\text { par chaque résident, au long des deux } \\
\text { ans, dans tous les contextes de presta- } \\
\text { tion de soins. } \\
\text { Un stage de médecine familiale qui fa- } \\
\text { cilite et favorise le suivi de clientèle. }\end{array}$ \\
\hline La cohérence & $\begin{array}{l}\text { L'exposition des résidents à un modèle de } \\
\text { pratique idéal qui répond aux besoins de la } \\
\text { population les prédispose à pratiquer dans de } \\
\text { tels modèles ou à mettre en place de telles } \\
\text { structures dans leur pratique future. }\end{array}$ & $\begin{array}{l}\text { Transformation des unités de médecine } \\
\text { de famille en milieux modèles de pres- } \\
\text { tation de soins. }\end{array}$ \\
\hline La réflexion & $\begin{array}{l}\text { L'apprentissage réflexif soutient le dévelop- } \\
\text { pement d'une pensée critique qui s'autono- } \\
\text { mise progressivement et favorise la méta- } \\
\text { cognition et le traitement plus en profondeur } \\
\text { de l'information. } \\
\text { La recherche et la réflexion sur nos pra- } \\
\text { tiques génèrent de nouvelles connaissances, } \\
\text { limitent l'empirisme et améliorent la qualité } \\
\text { de la pratique. }\end{array}$ & $\begin{array}{l}\text { Nouveau programme d'enseignement } \\
\text { formel réflexif inspiré des principes du } \\
\text { socioconstructivisme. Création d'un } \\
\text { réseau de recherche des Unités de mé- } \\
\text { decine de famille. } \\
\text { Journée annuelle de présentation des } \\
\text { travaux d'érudition et de recherche des } \\
\text { résidents }\end{array}$ \\
\hline
\end{tabular}

CRC auprès de diverses instances politiques, universitaires, organisationnelles et populationnelles.

Tout au long de cette démarche, le CReR s'est inspiré d'un projet pilote réalisé depuis 1998 au sein même de notre programme, dans l'UMF Notre-Dame du centre hospitalier universitaire de Montréal et dans celle du centre local de services communautaires (CLSC) des Faubourgs. Ce projet pilote, né d'une insatisfaction qu'exprimaient alors les résidents à propos de certains de leurs stages (chirurgie, gynécologie, unité d'enseignement de médecine interne), consistait à transformer partiellement le programme local de ces deux UMF en remettant en cause les stages qui posaient problème dans leur forme traditionnelle, à savoir celle d'une période de quatre semaines consécutives dans une 
même discipline. Dans la mesure où l'exposition clinique était jugée pertinente pour la formation d'un futur médecin de famille, la partie ambulatoire des stages en cause était conservée, voire amplifiée et intégrée dans un stage de médecine familiale allongé, qui passait ainsi de six à 12 périodes de quatre semaines. Ce programme longitudinal s'inspirait d'un modèle analysé dans le rapport du comité sur le curriculum de la section des enseignants du CMFC ${ }^{[2]}$. Selon ce rapport, un tel cursus longitudinal, en offrant aux résidents de médecine familiale un stage clinique centré sur leurs apprentissages, en privilégiant le contexte ambulatoire et en se rapprochant davantage du mode de pratique d'un médecin de famille en exercice, avait l'avantage de favoriser la transférabilité des aptitudes, la continuité des soins, l'exposition aux modèles de rôles et l'évaluation des résidents.

L'évaluation de ce projet, réalisée trois années après son implantation, a fait l'objet d'un rapport remis en avril 2002 à la direction du programme de résidence. En allongeant le temps passé en UMF et en améliorant la pertinence de l'exposition clinique, il était estimé que ce cursus longitudinal : augmentait la satisfaction que les résidents avaient de leur formation; donnait l'opportunité aux résidents d'être confrontés à une clientèle typique de celle d'un médecin de famille, constituée d'un nombre plus importante de patients, à laquelle ils offraient une meilleure disponibilité ; intensifiait l'exposition des résidents à des modèles de rôle de médecins de famille; favorisait la maturation professionnelle des résidents ; facilitait la transférabilité des apprentissages acquis par une exposition en médecine spécialisée dans un contexte de médecine de première ligne. Les auteurs du rapport documentaient comme difficultés : la lourdeur de la gestion des horaires pour les chefs d'UMF; une augmentation problématique des déplacements qu'imposait aux résidents la diversification de l'exposition clinique et une discontinuité dans la supervision des activités clinique qui compliquait la construction de l'évaluation. Les objectifs du nouveau programme étaient donc de conserver ces retombées positives identifiées et de tenter d'apporter une solution raisonnable aux difficultés recensées lors de ce projet pilote.

\section{Orientations du nouveau programme}

Les orientations du nouveau programme ont été définies à partir d'une réflexion concernant à la fois l'identification les besoins de santé de la population à desservir par les futurs médecins de famille diplômés et la définition de principes directeurs pédagogiques (tableau II).

Identification des besoins de santé

de la population à desservir

par les futurs diplômés

Les principaux besoins pris en compte ont été identifiés en s'appuyant sur plusieurs rapports et documents officiels disponibles ${ }^{[3-5]}$. Ils concernent notamment :

- Le besoin d'un suivi médical global et continu par un médecin de famille. Le nouveau programme allait devoir soutenir le développement du sens de responsabilité des résidents, en les stimulant pour qu'ils manifestent leur professionnalisme par un engagement soutenu à offrir des soins globaux et continus à une clientèle définie de patients.

- Les besoins reliés à la démographie. Le nouveau programme allait devoir préparer les résidents à offrir des services à une population vieillissante qui exige des soins complexes pour des maladies chroniques et multiples, tant en première ligne, en contexte ambulatoire et à domicile, qu'en seconde ligne, en centre hospitalier de soins de courte et de longue durée.

- Les besoins reliés aux difficultés d'accès aux services d'un médecin de famille. Le nouveau programme allait devoir s'inspirer du système québécois de santé dans ses efforts pour compenser l'impact de la pénurie d'effectifs médicaux par le travail interdisciplinaire et une organisation plus efficiente des structures de prestation de soins. 
Les principes directeurs pédagogiques

\section{La signifiance identitaire et le principe $d$ 'un enseignement et $d$ 'un apprentissage contextualisés}

La médecine familiale ne se définit plus comme résultant d'une absence de spécialisation ou, à l'inverse, comme constituant la somme des caractéristiques de base de toutes les autres spécialités. Il est maintenant bien établi et largement accepté que la médecine familiale est une discipline spécifique qui, parce qu'elle s'appuie sur un ensemble défini et spécifique de savoirs, d'habiletés et d'attitudes, parce qu'elle développe de nouveaux savoirs grâce à une recherche propre, actuellement en développement croissant, et parce qu' on y accède à l'issue d'un programme de formation postdoctorale et d'un processus de certification, constitue une spécialité ${ }^{[6,7]}$ en tant que telle. Nos collègues spécialistes d'organe ou de fonction ont un rôle important à jouer dans la formation de nos résidents mais il est illusoire de penser que l'on puisse devenir médecin de famille, spontanément, simplement après avoir terminé une succession de stages auprès d'internistes, de chirurgiens, de gynécologues, etc. Parce que c'est en médecine familiale que s'apprennent les principes de prestations de soins globaux et continus, tant préventifs que curatifs, ayant comme objectifs la promotion, le maintien ou le rétablissement du bien-être physique et psychique de patients de tous âges, l'importance de l'influence qu'ont la culture, la famille et la communauté sur la santé et la maladie et l'importance de répondre aux besoins de la communauté, c'est en médecine familiale, au contact des enseignants que sont les médecins de famille et leurs patients, que les résidents ont les meilleures chances de développer leur compréhension de la discipline. Puisqu'une exposition à des tâches authentiques, complexes et signifiantes, outre qu'elle stimule la motivation à apprendre, favorise la construction de l'identité professionnelle et facilite le développement d'un sentiment de compétence ${ }^{[8]}$, c'est en vivant, dans l'UMF, l'expérience d'une pratique qui ressemble à celle d'un médecin de famille en exercice que les résidents sont en situation la plus favorable pour construire leur identité de médecin de famille et développer leurs stratégies de reconnaissance et de traitement des problèmes de santé de première ligne ${ }^{[2,9]}$. Comme le résument bien Rat et al. ${ }^{[10]}$, en constatant que le niveau de développement professionnel de leurs internes finissants est plus abouti chez ceux qui ont fait un stage chez le praticien et un stage ambulatoire en soins primaires en autonomie supervisée, «c'est en faisant de la médecine générale que l'on devient généraliste ».

Le CReR a donc voulu, conformément aux prescriptions d'agrément du CMFC ${ }^{[11]}$, que le nouveau programme concentre la plus grande partie des activités de nos résidents dans les UMF et dans le réseau de soins de première ligne, afin que l'expérience pratique et authentique en médecine familiale serve d'assise à la formation et que les médecins de famille jouent un rôle prépondérant dans l'enseignement. En vertu de ce principe et en cohérence avec la perspective de l'enseignement et de l'apprentissage contextualisés authentiques ${ }^{[12]}$, la médecine familiale est devenue le contexte principal et continu d'apprentissage, les médecins de famille en sont devenus les principaux enseignants et la durée du stage de médecine familiale est passée de 6 à 15 périodes sur les 26 périodes que compte la formation.

\section{L'itération}

Plutôt que la réussite successive d'une suite de stages, c'est la répétition de l'exposition clinique tout au long des deux années de formation qui est considérée comme étant le moyen privilégié de construction des compétences ${ }^{[8]}$. L'élimination de certains stages de spécialité qui avaient un faible rendement pédagogique a totalement modifié l'architecture du programme. Le nouveau cursus, plus longitudinal, fait maintenant une large place au stage de médecine familiale, à l'intérieur duquel diverses expositions cliniques, ciblées pour leur pertinence, se répètent périodiquement, tant à l'intérieur que hors des UMF, pendant les deux années de la formation, favorisant ainsi un apprentissage en 

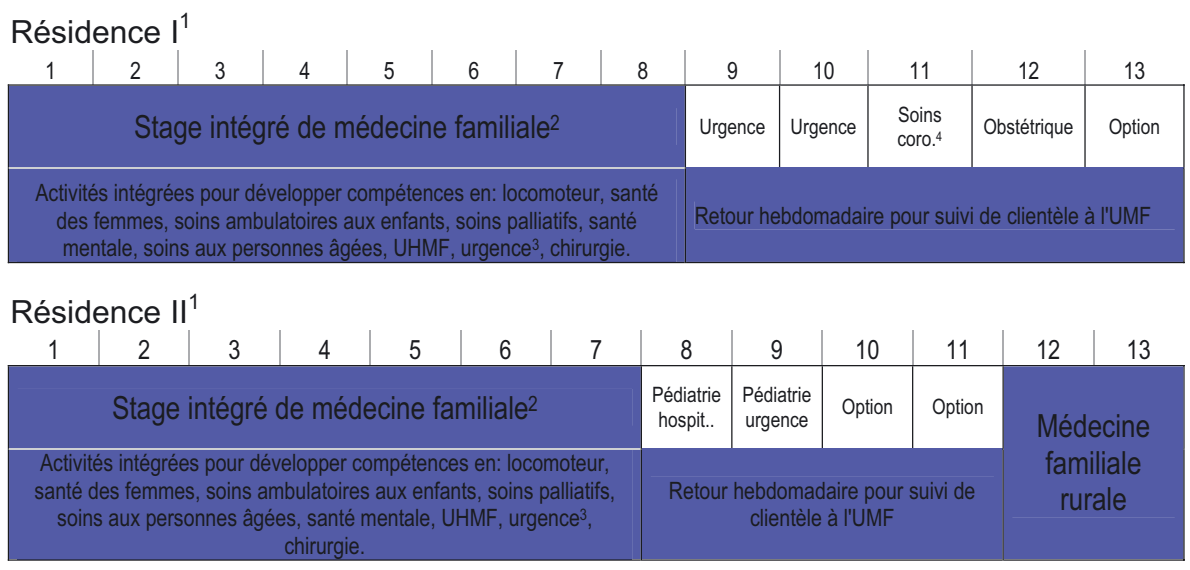

1: La séquence des stages peut varier d'une UMF à l'autre.

$2: 50 \%$ du temps doit être passé en continuité de soins.

$3: 16$ quart de travail, jour-soir-nuit.

4 : Cardiologie ou soins intensifs adaptés à la première ligne.

Fig. 1. Maquette de stage du programme de résidence en médecine familiale de l'Université de Montréal.

profondeur. Dans cette nouvelle structure, nos résidents sont appelés à effectuer un travail clinique qui s'apparente beaucoup plus à celui d'un médecin de famille en exercice.

\section{La responsabilisation}

Pour amener les résidents à s'investir en professionnels accomplis dans le suivi continu de leurs patients, le nouveau programme met l'accent sur la continuité dans tous les contextes de soins, tant de première que de deuxième ligne et ce, tout au long des deux années de la formation. Tout en faisant le pari qu'une exposition prolongée en médecine familiale favorisera et facilitera le suivi de clientèle, le nouveau programme, comme le faisait l'ancien, a conservé la politique de confier à chaque résident, dès son entrée en formation, une clientèle de patients pour laquelle il devient le médecin de famille et du suivi médical de laquelle il est responsable, sous la supervision de médecins de famille enseignants, tout au long des deux années de la formation.

\section{La cohérence}

Il est apparu essentiel que nos résidents soient formés dans des structures de prestation de soins qui ressemblent le plus possible à celles dans lesquelles ils seront appelés à travailler une fois leur formation terminée. Une organisation exemplaire de prestation de soins, appelée à représenter pour les résidents un modèle idéal de pratique, devrait soit les préparer adéquatement à travailler dans de telles organisations, soit, si elles n'existent pas, les inciter à contribuer à les concevoir. À cette fin, la direction du programme a demandé à toutes les UMF de se transformer en cliniques modèles qui intègrent, à côté des médecins, d'autres professionnels (infirmières, psychologues, pharmaciens, ...) appelés à contribuer tant à l'enseignement qu'à la prestation de soins en interdisciplinarité.

\section{La réflexion}

Le CReR a voulu que le nouveau programme inculque aux résidents des habitudes de pratique réflexive et suscite un intérêt pour la recherche sur les activités cliniques et pédagogiques exercées en première ligne par des médecins de famille. Ceci s'est traduit par la conception d'un nouveau programme d'enseignement valorisant la réflexion et visant à favoriser la mobilisation et l'intégration des ressources des résidents, par l'institutionnalisation d'une journée annuelle de présentation de travaux d'érudition et de recherche des résidents et par la création d'un réseau de recherche dans les UMF. 


\section{La construction des compétences}

La faculté de médecine de l'Université de Montréal a pris la décision, il y a dix ans déjà, de faire basculer la formation de ses étudiants d'une approche pédagogique par objectifs à une approche pédagogique par compétences. Cette décision n'a pas encore eu de répercussion pratique au niveau de la formation post-graduée (troisième cycle) mais elle est exploitée, dans notre programme, pour stimuler les résidents à prendre conscience du rôle actif qu'ils doivent jouer dans leur processus d'apprentissage et pour les inciter à le faire. Parce qu'il adhère totalement aux principes du paradigme d'apprentissage $^{[13]}$, fortement convergents avec l'approche pédagogique par compétences ${ }^{[14]}$, notre programme compte collaborer étroitement avec les instances facultaires pour réaliser cette bascule qui se concrétisera progressivement au cours des prochaines années. Les quatre principes par lesquels le CMFC ${ }^{[11]}$ définit la médecine familiale et les rôles CanMEDS ${ }^{[15]}$ serviront de guide pour la construction d'un répertoire de compétences, première étape de cette transformation attendue.

\section{Le processus de révision curriculaire mis en œuvre}

Le processus de révision curriculaire a d'abord comporté une démarche intégrative des activités d'enseignement dans le cadre des stages. Il a aussi exploité certains outils de communication. Enfin, il s'est adossé sur une gouvernance stratégique.

\section{Une démarche intégrative des activités}

d'enseignement dans le cadre des stages

Les besoins de formation ayant été identifiés et les principes directeurs pédagogiques énoncés, le CReR a élaboré un processus de révision curriculaire permettant à la fois de soutenir l'affirmation de la spécificité de notre discipline et de se réapproprier son contenu, son contrôle et son enseignement. Il s'inspire des orientations déterminées par la direction du programme et se réalise, dans chaque UMF, en quatre étapes présentées ici, pour des raisons didactiques, dans une chronologie plus rigide qu'elle ne l'est en temps réel.

- La première étape consiste à supprimer les stages à faible rendement pédagogique, considérés comme tels soit parce que les activités d'enseignement y sont insuffisantes, soit parce que l'exposition clinique y est moins pertinente pour un futur médecin de famille dans le contexte d'une courte formation de deux ans. Il s'agit essentiellement, pour notre programme, de stages réalisés en milieu hospitalier, dans les services de spécialités.

- La deuxième étape consiste à utiliser les périodes ainsi libérées pour allonger le stage de médecine familiale. Celui-ci a pu passer, graduellement, de six périodes, durée qu'il occupait dans l'ancien programme, à 15 périodes dans le nouveau programme.

- La troisième étape consiste à identifier, pour se les approprier, les compétences à construire dans chacun des domaines qui faisaient auparavant l'objet d'un stage dont nous avions délégué la gouvernance pédagogique aux spécialistes.

- La dernière étape, enfin, consiste à mettre en œuvre une nouvelle stratégie pédagogique qui planifie une exposition clinique itérative, centrée sur les besoins d'un médecin de famille et sollicitant un partage des responsabilités d'enseignement entre médecins de famille et médecins spécialistes. Conformément aux principes évoqués plus haut, l'exposition clinique se fait principalement en médecine familiale et l'enseignement est assuré principalement par des médecins de famille. La collaboration avec les spécialistes enseignants, dont la contribution pédagogique est toujours valorisée et recherchée, se fait maintenant en vertu d'un nouveau partenariat. Celuici consiste à leur confier nos résidents en leur demandant de leur enseigner non plus ce qu'ils jugent pertinent qu'un médecin de famille apprenne dans leur propre domaine d'expertise de spécialiste, mais ce qui est spécifiquement identifié dans le cadre du programme de résidence de 
médecine familiale, en leur proposant en outre une durée et une fréquence de répétition de l'exposition clinique.

\section{Des outils de communication}

\section{Le cahier du programme}

La direction du programme, avec le support du $\mathrm{CReR}$, a produit un document-cadre qui comporte un répertoire de compétences à construire par secteurs de soins, pour en faire un outil de référence à utiliser, notamment, lors des deux dernières étapes du processus d'intégration.

\section{Le guide à l'intention des responsables d'UMF de l'Université de Montréal}

Ce document de référence, élaboré par la direction du programme, clarifie, explicite et uniformise les politiques du programme et les divers processus administratifs mis en œuvre dans la gestion d'une UMF. Il constitue un réel cahier des charges permettant aux responsables d'harmoniser les structures et les pratiques de leur UMF avec les prescriptions des organismes d'agrément, les règlements facultaires et les orientations du programme.

\section{Une gouvernance stratégique}

Un plan central de transformation sur trois ans a finalement été établi et placé sous la gouvernance de la direction du programme. Chaque UMF a reçu le mandat d'élaborer son plan local de transformation, qui respecte et s'inspire du plan central en prévoyant, en fonction des ressources et opportunités locales d'enseignement, les modalités d'évolution de l'ancien vers le nouveau programme.

\section{Le nouveau programme de résidence de médecine familiale}

Le nouveau programme articule des activités de formations liées à l'exposition clinique durant les périodes de stage (figure 1) et des activités d'enseignement théorique.

\section{L'exposition clinique}

Le stage intégré de médecine familiale constitue le cœur de la formation et accapare 15 des 26 périodes que comporte le programme.

Pour des raisons de pertinence pédagogique, certains stages ont conservé leur forme dite concentrée, qui consiste en quatre semaines consécutives de travail dans une même discipline. Ce sont :

- En début de résidence, pendant la première moitié de la première année : une période d'urgence, une période de soins intensifs ou de soins coronariens et une période d'obstétrique.

- En milieu de résidence, entre la moitié de la première année et la moitié de la deuxième année : une période de gériatrie, une période d'urgence pédiatrique et une période d'hospitalisation pédiatrique.

- En fin de résidence, durant la deuxième année : deux périodes contiguës de médecine familiale rurale. Il s'agit d'un stage de médecine générale en région éloignée.

- À n'importe quel moment durant la formation : trois périodes d'options au choix du résident.

Durant le stage intégré de médecine familiale, le résident doit employer 40 à $50 \%$ de son temps à effectuer des activités cliniques de suivi de clientèle, principalement à l'UMF, en consultation avec et sans rendez-vous, mais aussi à domicile et en centre d'hébergement. Ce travail clinique lui permettra d'assurer la continuité attendue des soins à sa clientèle. Le reste du temps clinique est consacré à des activités intégrées qui se répéteront périodiquement tout au long des deux ans de la formation, à savoir : huit semaines au sein d'unités d'hospitalisation en médecine familiale, 32 à 40 quarts de travail en service d'urgence et une variété d'activités qui permettront de construire les compétences attendues en pathologie de l'appareil locomoteur, santé des femmes, pratique ambulatoire de la pédiatrie, 
pratique ambulatoire, hospitalière et à domicile des soins palliatifs, santé mentale et chirurgie.

\section{L'enseignement théorique}

En complément de l'exposition clinique, un enseignement théorique organisé dans le cadre d'un programme d'enseignement formel, commun à tous les résidents, enrichit la formation. Ce programme d'environ 300 heures, planifié dans le cadre d'un cycle de deux ans, valorise l'enseignement réflexif et comporte :

- des journées académiques qui regroupent à l'Université, trois fois par an, tous les résidents du programme pour participer à des séminaires sur des sujets variés tels que : l'examen médical périodique, le suivi obstétrical, la médecine du travail, l'éthique, etc.

- Une série de cours, sous forme de discussion de cas cliniques en petits groupes de huit à dix résidents avec un enseignant facilitateur, en utilisant une approche conforme au «Programme d'apprentissage en petits groupes basé sur la pratique » $(\mathrm{PGBP})^{[16]}$, développé par la Fondation pour l'éducation médicale continue en collaboration avec l'Université McMaster. Ces discussions de cas d'une durée d'environ 90 minutes se tiennent, hebdomadairement, dans les UMF.

- Une série d'ateliers portant sur : la communication médecin patient, selon le modèle CalgaryCambridge; l'éthique en médecine; la gestion critique de l'information enseignée dans le cadre de modules d'auto-apprentissage des habiletés de lecture critique et de gestion de l'information, tels qu'ils ont été créés à l'Université Laval ${ }^{[17]}$, et du club de lecture se tenant dans chaque UMF; une formation de base à la pédagogie.

- Une formation en soins critiques périnataux d'une durée de trois jours, qui incorpore la réanimation néonatale et les urgences obstétricales.

- Une formation sur le système de santé québécois.

Tous les résidents, dans chacune des UMF, sont pourvus d'un tuteur qui a pour mandat de faire office d'accompagnateur pédagogique. Il appartient à ce dernier de faciliter pour ses tuteurés l'articulation entre l'apprentissage pratique et l'enseignement théorique. Puisque le tuteur est aussi un enseignant de l'UMF qui travaille étroitement avec ses tuteurés, il accomplit cette tâche par le biais d'observations cliniques répétées, dans divers contextes de soins, et grâce à des entretiens périodiques.

\section{Évaluation préliminaire du nouveau dispositif}

Des impacts positifs recueillis auprès des résidents

Convaincu de la nécessité d'apprécier l'impact des changements apportés au programme de formation, le CReR s'est adjoint, dès 2007, le concours d'un agent de recherche et d'un médecin de famille enseignant ayant toutes deux une solide expérience en recherche pour concevoir l'évaluation du nouveau programme. Limité dans ses moyens, le CReR a fait les choix suivants :

- Cibler deux axes d'évaluation en cohérence avec l'objectif principal du nouveau programme (préparer ses résidents à répondre adéquatement aux besoins de santé de notre population), en questionnant : a) la perception qu'ont les résidents de leur compétence dans les domaines de soins qui font l'objet d'intégration dans le stage de médecine familiale; b) l'intérêt des résidents à s'engager auprès d'une clientèle déterminée de patients à offrir une continuité des soins.

- Débuter l'exercice d'évaluation dès le début de l'implantation du nouveau programme pour que l'information obtenue permette d'apporter les correctifs nécessaires au fur et à mesure des changements.

- Obtenir l'information annuellement, en fin d'année académique, par le biais de questionnaires anonymes remis à tous les résidents finissants de toutes les UMF du programme.

Les questionnaires de la première année, remplis par les finissants de la cohorte 2006-2008, portaient sur 
les secteurs de soins qui devaient être intégrés prioritairement, c'est-à-dire : les soins aux enfants, la santé des femmes et la pathologie de l'appareil locomoteur. Les résultats obtenus doivent être interprétés avec prudence puisqu'à ce moment plusieurs UMF avaient à peine commencé à transformer leur programme local. Avec un taux de réponse très satisfaisant de $89 \%$, les questionnaires ont révélé que :

- les résidents expriment un sentiment de compétence élevé : $80 \%$ des répondants s'estiment compétents pour offrir des soins de qualité dans les secteurs étudiés ;

- l'unité de médecine familiale est perçue par les résidents comme l'un des lieux les plus utiles à l'apprentissage dans les trois secteurs concernés. Son intérêt est jugé supérieur à celui des autres lieux d'apprentissage que sont les services de consultations externes, les unités hospitalières de soins, la salle d'urgence ;

- lorsque, dans certains domaines spécifiques de ces secteurs de soins, le sentiment de compétence est faible, ni la qualité de l'enseignement, ni la compétence des médecins de famille enseignants ne sont en cause. Les résidents attribuent principalement au manque d'exposition clinique la raison de la non-maîtrise de certaines capacités ;

- l'intérêt pour la continuité de soins est relativement faible : seuls $60 \%$ des répondants manifestaient le désir d'inclure dans leur pratique future cette facette de la pratique.

L'exercice d'évaluation en est encore à ses tout premiers débuts. Les résultats obtenus ne nous permettent certainement pas de porter un jugement quant à l'atteinte des objectifs de la réforme du programme, ni la valeur ajoutée des changements apportés à l'ancien programme. Ils proviennent d'une première cohorte qui n'a été exposée qu'à une partie des changements planifiés. Il faudra attendre que toutes les UMF aient achevé la transformation de leur programme local, dans quelques années, avant de porter un jugement global. Entre temps, ces premiers résultats sont rassurants et permettent déjà d'apporter certains correctifs au processus en cours.
Des nouveaux défis à relever

Notre nouveau programme s'implante progressivement dans nos UMF depuis bientôt trois ans. Bien que le CMFC l'ait trouvé suffisamment achevé pour lui accorder un agrément complet de six ans lors de sa visite d'avril $2008^{[18]}$, sa mise en œuvre nous confronte, de façon quasi quotidienne, à de nombreux défis.

Puisque la formation des résidents est concentrées sur les ressources de médecine familiale et que nous faisons des médecins de famille les enseignants principaux de notre programme, il est impératif d'obtenir, pour nos UMF, les ressources financières, humaines et matérielles qui leur permettront de réaliser les transformations attendues d'elles et d'offrir à notre corps professoral les outils nécessaires au rehaussement de leurs compétences clinique et pédagogique. L'obtention de ces ressources est une condition sine qua non de réussite de la transformation du programme. La direction du département, l'équipe de direction du programme et celle de chaque UMF y consacrent une part importante de leurs énergies.

L'organisation de l'exposition clinique dans le stage intégré de médecine familiale pose, au-delà de l'énorme défi de la gestion des horaires et des évaluations, celui de l'équilibre entre le global et le spécifique, le continu et le fragmenté. Quelle place faut-il accorder aux incontournables cliniques thématiques (ORL, dermatologie, pathologie de l'appareil locomoteur...) qui, tout en enrichissant de façon souhaitable l'éventail de l'exposition clinique, sont susceptibles d'entraîner, avec de nombreux déplacements physiques, un morcellement et une dispersion de l'apprentissage ? La recherche de cet équilibre fait encore l'objet de réflexions et d'échanges entre les divers intervenants du programme.

L'évaluation des résidents reste au cœur de nos préoccupations. La portion du stage intégré de médecine familiale passée hors UMF avec les spécialistes nous pose le problème de la conception d'une évaluation fiable du travail que font nos résidents lorsque nous ne pouvons les observer. Sans 
doute, la solution à ce problème réside-t-elle dans l'adoption de nouvelles stratégies d'évaluation qui s'inspireront des principes pédagogiques du paradigme d'apprentissage.

\section{Conclusion}

Née d'un besoin tant ressenti que démontré, la transformation de notre programme de résidence s'est réalisée en respectant une démarche rigoureusement planifiée et soigneusement gérée. Le choc des idées qu'elle a provoqué, la mobilisation d'énergie créatrice qui en a résultée et la mise en place d'innovations pédagogiques se sont déjà révélés plus féconds que ne le laisse paraître une certaine impression de désordre inhérente à tout dispositif encore en chantier.

Le sentiment que nous avons de cheminer dans la bonne direction doit, bien sûr, être vérifié. Aussi, la nécessaire évaluation de l'impact qu'auront sur la formation de nos résidents les transformations du programme a d'ores et déjà été simultanément entreprise. Nous souhaitons qu'elle apporte une réponse claire à la question fondamentale de savoir si nos résidents, au bout du compte, seront mieux préparés pour répondre adéquatement aux besoins de notre société. Elle nous permettra, à tout le moins, de corriger le tir en cours de route pour que notre programme forme des médecins de famille hautement compétents.

\section{Contributions}

Gilbert Sanche est le principal auteur et rédacteur de l'article. Louise Authier, Caroline Béique et Réjean Duplain ont contribué au contenu intellectuel de l'article en participant activement à sa rédaction et à sa révision.

Le contenu de cet article a fait l'objet d'une communication de l'auteur principal lors du $8^{\mathrm{e}}$ Congrès du Collège national des généralistes enseignants (CNGE), qui s'est tenu à Angers (France) le 28 novembre 2008.

\section{Références}

1. Comité de révision du curriculum. Rapport du comité de révision du programme de résidence en médecine familiale de l'Université de Montréal. Novembre 2001 (Disponible sur demande auprès de l'auteur).

2. Le Collège des médecins de famille du Canada. Le curriculum postdoctoral de médecine familiale : une approche intégrée. Rapport du comité sur le curriculum de la section des enseignants. 1995. [On-line] Disponible sur : http://www.cfpc.ca/ French/cfpc/education/home/default . asp?s=1

3. Institut national de santé publique Québec, MSSS Québec. Portrait de santé du Québec et de ses régions 2006. [On-line] Disponible sur : http: //www . inspq.qc.ca/ pdf/publications/portrait_de_sante.asp

4. Ministère de la santé et des services sociaux du Québec. Plan de la santé et des services sociaux. Pour faire les bons choix. Québec : Bibliothèque Nationale du Québec. 2002 [On-line] Disponible sur : http://msssa4. msss.gouv.qc.ca/fr/document/publication . nsf/961885cb24e4e9fd85256b1e00641a29/ d5c257cf903fbeed85256c7500692d64? OpenDocument

5. Collège des médecins du Québec. Nouveaux défis professionnels pour le médecin des années 2000. Commission sur l'exercice de la médecine des années 2000. Montréal, 1998.

6. Bailey T. La médecine familiale est-elle une spécialité ? Can Fam Physician 2007;53:225-7.

7. Wonca Europe. La définition européenne de la médecine générale - médecine de famille. 2002. [On-line] Disponible sur : http://www. cnge.fr

8. Lasnier F. Réussir la formation par compétences. Montréal : Guérin, 2000.

9. Reust CE, Stehney M. Models of innovation : Longitudinal Curriculum in Family Practice residency Education. Leawood (Ks): Society of Teachers of Family Medicine, 2001.

10. Rat C, LeMauff P, VanWassenhove L, Goronflot L, UrionLaclaille J, Senand R. Diplôme d'études spécialisées de médecine générale. La validation des premiers internes à Nantes. Exercer 2008;81:45-8.

11. Le Collège des médecins de famille du Canada. Critères pour l'agrément des programmes de résidence : Médecine familiale, Médecine d'urgence, Compétences avancées en soins palliatifs. Mississauga (On) : Le Collège des médecins de famille du Canada. 2006. [Online] Disponible sur : http://www.cfpc.ca/French/ cfpc/education/accreditation/default.asp? $\mathrm{s}=1$ 
12. Frenay M, Bédard D. Des dispositifs de formation universitaire s'inscrivant dans la perspective d'un apprentissage et d'un enseignement contextualisés pour favoriser la construction de connaissances et leur transfert. In : A Presseau et M Frenay (dir.) : Le transfert des apprentissages. Québec : Les Presses de l'Université Laval, 2004.

13. Jouquan J, Bail P. À quoi s'engage-t-on en basculant du paradigme d'enseignement vers le paradigme d'apprentissage ? Exemple d'une révision curriculaire en résidanat de médecine générale. Pédagogie Médicale 2003;4:163-75.

14. Nguyen D-Q, Blais J-G. Approche par objectifs ou approche par compétences ? Repères conceptuels et implications pour les activités d'enseignement, d'apprentissage et d'évaluation au cours de la formation clinique. Pédagogie Médicale 2007;8:232-51.

15. Frank JR. (Ed). The CanMEDS 2005 physician competency framework. Better standards. Better physicians. Better care. Ottawa: The Royal College of Physicians and Surgeons of Canada, 2005. [On-line]
Disponible sur: http://crmcc.medical.org/ canmeds/CanMEDS2005/index . php

16. La fondation pour l'éducation médicale continue. Programme d'apprentissage en petit groupe basé sur la pratique (PGBP). [On-line] Disponible sur http:// www . fmpe.org/fr/programs/pbsg.html

17. Département de médecine familiale, Faculté de médecine, Université Laval. Les modules d'autoapprentissage des habiletés de lecture critique et de gestion de l'information. [On-line] Disponible sur : http://infocritique. fmed.ulaval.ca/Cours/ infocritique/index. aspx

18. Collège des médecins de famille du Canada et Collège des médecins du Québec. Rapport de l'équipe d'agrément, programme de résidence en médecine familiale. Avril 2008. Disponible sur demande auprès de l'auteur.

Correspondance et offprints : Gilbert Sanche, 1351 boul. des Laurentides, Laval, Québec, Canada, H7L 4W1 ; (450) 837-4276.

Mailto : gilbert.sanche@umontreal.ca 